\title{
Testing the GAD throughout geological time
}

\author{
M. E. Evans and G. S. Hoye \\ Institute for Geophysical Research, University of Alberta, Edmonton, Alberta, Canada T6G 2G7 \\ (Received October 20, 2006; Revised February 11, 2007; Accepted February 13, 2007; Online published July 20, 2007)
}

\begin{abstract}
The suggestion that the morphology of the geomagnetic field can be tested by inspecting the statistical distribution of palaeomagnetically-determined inclination angles has been challenged on the grounds that typical plate velocities require extremely long sampling intervals - perhaps exceeding the age of the Earth-in order to adequately sample the Earth's surface. Here, we investigate this problem by means of a random walk model and conclude that an interval equivalent to Phanerozoic time (600 Myr) is generally sufficient.
\end{abstract}

Key words: Geocentric axial dipole, zonal geomagnetic fields, random walk model.

\section{Introduction}

The general morphology of the geomagnetic field is of fundamental importance to palaeomagnetism and geomagnetism. In the former, the assumption of a dipole model is a cornerstone for all discussions concerning plate reconstructions. In the latter, it is the attempt to understand the complex dynamics of the earth's interior that is of interest. Historically, the idea of a dipolar geomagnetic field began with the publication of William Gilbert's De Magnete in 1600 , and was placed on a firm mathematical foundation by Carl Friedrich Gauss in his Allgemeine Theorie des Erdmagnetismus, published in 1839. Despite this long history of enquiry, it has been very difficult to actually demonstrate whether or not the dipolar morphology has been dominant throughout geological time.

Runcorn (1954) argued that the Coriolis force dominates fluid flow in the Earth's core and that the time-averaged geomagnetic field should therefore be symmetrical about the spin axis. In a subsequent paper (Runcorn, 1959), he considered the possibility that axially symmetric multipole fields (quadrupole, octupole etc.) might have existed for considerable parts of geological time, rather than a simple geocentric axial dipole (GAD). Despite the paucity of results then available, he was able to muster 13 relevant palaeomagnetic poles (Cambrian, Carboniferous, Permian, and Triassic for Australia, Europe, and North America, plus Carboniferous for South Africa). With the continents fixed in their present positions, he demonstrated that the colatitudes implied by these results do not support any of the pure zonal models he considered (dipole, quadrupole, octupole). He concluded that continental drift is a "more satisfactory inference". In retrospect, this is perhaps not surprising; no one now seriously doubts the reality of continental drift. But it was not always so. In fact, the pioneering work of Runcorn himself was of paramount importance in establishing the new orthodoxy. The problem is that the ac-

Copyright (c) The Society of Geomagnetism and Earth, Planetary and Space Sciences (SGEPSS); The Seismological Society of Japan; The Volcanological Society of Japan; The Geodetic Society of Japan; The Japanese Society for Planetary Sciences; TERRAPUB. ceptance of relative movement between continental blocks means that Runcorn's 1959 analysis allows no conclusions to be drawn about the morphology of the field, GAD or otherwise. If one assumes a field model, the relative movements of the continents can be constrained, but the validity of the field model cannot be independently checked.

Towards the end of his 1959 paper, Runcorn appeals to palaeoclimatic evidence as a possible independent source of relevant information - an idea that had already been discussed by Irving some years earlier (Irving, 1956). Blackett (1961, 1962) also took up this idea and Briden and Irving (1964) systematically compiled the geographic distribution of such features as glacial deposits and coral reefs for which palaeomagnetic results were available. The outcome was that the palaeolatitude spectra obtained were broadly consistent with a dipolar field. No statistical tests were performed. Indeed, it is difficult to see how this could be done without the luxury of a theoretical model for the expected distribution of any given palaeoclimatic indicator. As an illustration, consider glacial deposits. It is tempting to expect them to occur preferentially in high latitudes, but drastic past global changes-like Snowball Earth (Hoffman and Schrag, 2002), for example — quickly dispel any hope of devising a robust theoretical model. The same is true for all the palaeoclimatic indicators that have been proposed. General trends are probably reliable, but these cannot be quantified sufficiently to permit formal statistical tests.

Another suggestion-first put forward by R. L. Wilson (1961) — relies on the latitudinal dependence of the strength $(F)$ of the field at the Earth's surface. For a GAD field, this is given by

$$
F=\left(\mu_{0} / 4 \pi\right)\left(M / r^{3}\right)\left(1+3 \cos ^{2} \theta\right)^{1 / 2}
$$

where $\mu_{0}$ is the permeability of free space, $M$ is the dipole moment, $r$ is the Earth's radius, and $\theta$ is the colatitude. A global compilation of 266 palaeointensity results spanning the last 10 million years yields a latitudinal profile that "is concordant with a theoretical curve from a geocentric axial dipole" (Tanaka et al., 1995). This is certainly encouraging, but the severely restricted time span does not permit 
firm conclusions about more remote geological times. Perrin and Shcherbakov (1997) have made an extended compilation $(\mathrm{N}=879)$ covering the last 400 million years and conclude that it is also consistent with a dominantly GAD morphology. However, the use of palaeointensity data for testing long-term field morphology faces some intrinsic difficulties. The observations show a wide range of values, standard deviations of $\sim 50 \%$ being common. Some of this variance is no doubt due to experimental error, but the geomagnetic field itself does actually exhibit large, rapid fluctuations (McElhinny and Senanayake, 1982; Korte and Constable, 2005). When plotted against palaeolatitude, such wide bounds make it difficult to discriminate between rival field models. Furthermore, the palaeolatitudes themselves are obtained by assuming a dipole morphology.

A third proposal, involving the statistical distribution of observed palaeoinclination angles, was originally put forward some thirty years ago (Evans, 1976) and used to perform a formal statistical test on the pure zonal fields considered by Runcorn (1959). As more data became available, this concept was used to inspect sub-divisions of geological time (as opposed to the entire Phanerozoic considered in the original paper) and also to extend the analysis to Precambrian time (Piper and Grant, 1989; Kent and Smethurst, 1998). Furthermore, it became possible to assess more complicated magnetic morphologies containing mixtures of zonal harmonics (Kent and Smethurst, 1998), for which the original test works equally well, of course. More recently, Bloxham (2000) has made use of palaeoinclination distributions in his study of the sensitivity of geomagnetic field morphology to the growth of the inner core and to thermal interactions between the mantle and the core. For our present purposes, however, the most important developments involve the work of Meert et al. (2003) and McFadden (2004), who raise serious objections to the palaeoinclination test. Both papers conclude that the 600 million years represented by Phanerozoic time is too short to satisfy the underlying requirement that the Earth's surface be adequately sampled.

By modeling continental drift as a moving spherical cap, Evans (2005) was able to provide a counter-example to McFadden's conclusions, which were based on the idea of a single drifting point. However, the spherical cap procedure actually used had a serious drawback in that only one (very simple) drift trajectory was considered. This may have been a useful first step, but it provided no insight into the likelihood of success of the palaeoinclination test in general. Here, we adopt a random walk procedure, like that introduced by Meert et al. (2003), to investigate large numbers of drift histories.

\section{The Palaeoinclination Test}

In general, for any axial multipole field, the angle of inclination $(I)$ is given by

$$
\tan \left(I_{l}\right)=-(1+l) P_{l} /\left(\partial P_{l} / \partial \theta\right)
$$

where $P_{l}$ is the Legendre polynomial of degree $l$. For a GAD field $l=1$, giving the familiar expression

$$
\tan (I)=2 \cot (\theta)
$$

(a)

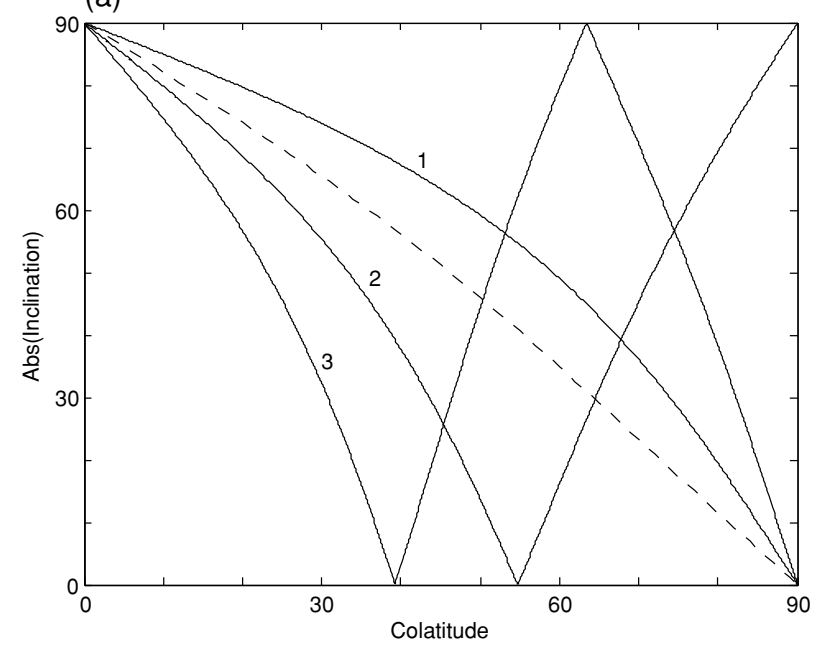

(b)

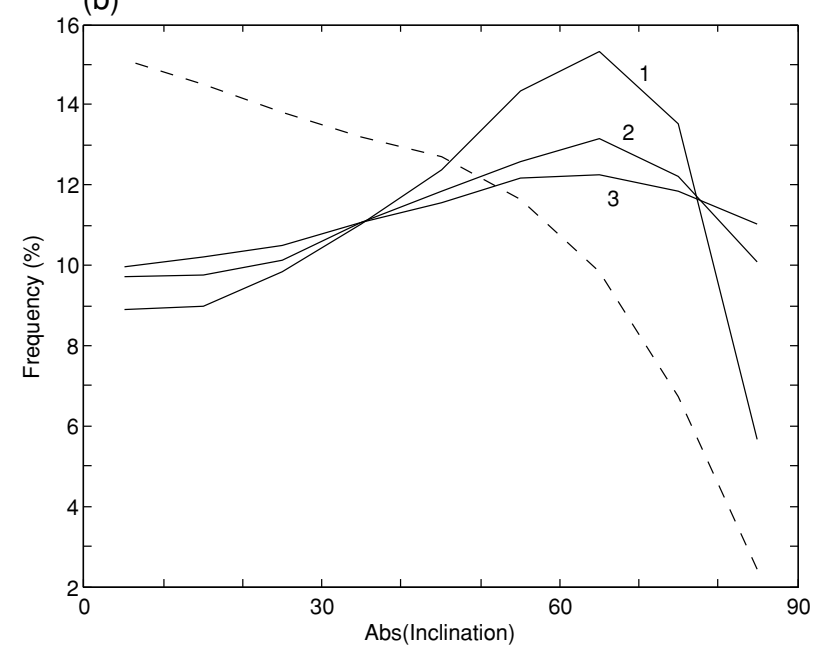

Fig. 1. (a) Inclination as a function of colatitude for zonal dipole $(l=1)$, quadrupole $(l=2)$, and octupole $(l=3)$ fields. The dashed curve indicates the effect of combining a unit dipole with a $20 \%$ octupole (i.e. $g_{3}^{0} / g_{1}^{0}=+0.2$ ). (b) Distributions of palaeoinclination angles for the models shown in (a). The sensitivity of the palaeoinclination test is particularly well illustrated by the marked effect of the dipole-octupole combination (dashed curve: $g_{3}^{0} / g_{1}^{0}=+0.2$ ).

Figure 1(a) indicates how the angle $I$ varies between the poles and the equator for $l=1$ (dipole), $l=2$ (quadrupole), and $l=3$ (octupole) fields. The effect of combining zonal harmonics, as suggested by numerous authors (e.g. Briden et al., 1970; Westphal, 1993; McElhinny et al., 1996; Kent and Smethurst, 1998; Torsvik and van der Voo, 2002), is illustrated by the dashed curve, for which $g_{3}^{0} / g_{1}^{0}=+0.2$. The proposed palaeoinclination test argues that if palaeomagnetic results are compiled for an interval during which sufficient plate motions have occurred, then specific distributions will be found. This is because each field morphology is associated with its own particular distribution (Fig. 1(b)). In the original publication (Evans, 1976), 1271 published palaeomagnetic results spanning the entire Phanerozoic (0-600 Ma) were analyzed. It was found that the observed distribution could not be statistically distinguished from a GAD field, whereas all higher-order fields failed the $\chi^{2}$ test.

The important question as to how long the sampling inter- 


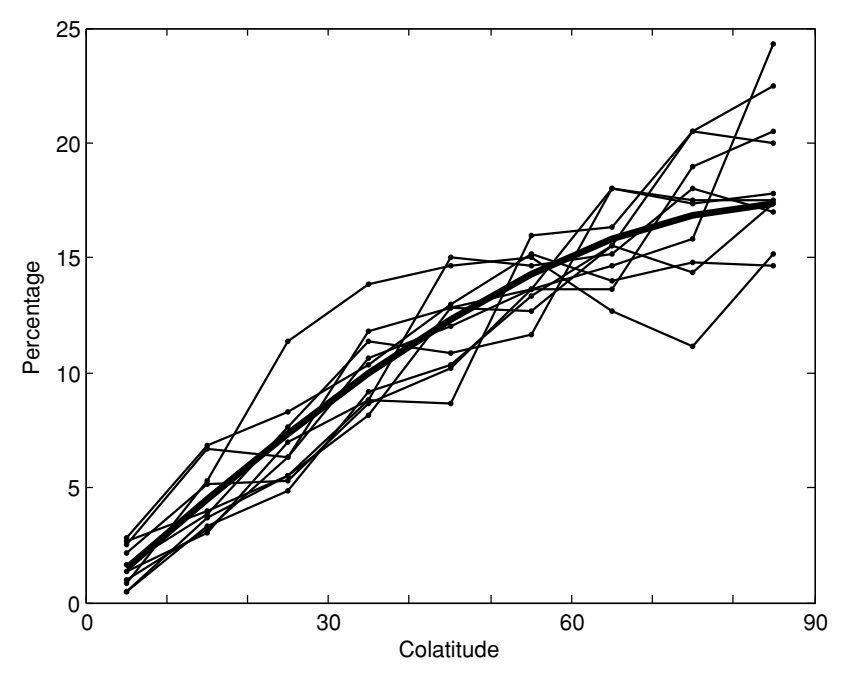

Fig. 2. Percentage of "sites" falling in $10^{\circ}$ colatitude bands for 10 separate $600 \mathrm{Myr}$ drift histories. The heavy curve indicates the theoretically expected distribution.

val should be in order to provide a suitable random sample of the globe's surface was raised by Meert et al. (2003) and by McFadden (2004). McFadden correctly points out that continents cannot simply disappear and reappear at will; they must actually drift over the Earth's surface in a continuous trajectory. He therefore considers a point drifting at a constant speed, $s$, in such a way that it spends a time in each latitude band proportional to the surface area represented by that band. Taking $s$ to be $6 \mathrm{~cm} / \mathrm{yr}$ (a typical plate speed) and considering $10^{\circ}$ latitude bands (as was done in the original 1976 analysis), he demonstrates that 2405 million years are necessary. If - as McFadden recommends $-5^{\circ}$ latitude bands are used, the time required exceeds the age of the Earth. However, these conclusions are greatly modified if one considers observations made on a drifting continent of finite size instead of a single drifting point. Evans (2005) considers a single continent in the form of a spherical cap (dubbed Capland) with a radius of $60^{\circ}$ (i.e. covering $25 \%$ of the Earth's surface, similar to the present land surface). As a preliminary test case, Capland was allowed to drift at uniform speed down a meridian of longitude from pole to pole and it was found - not unexpectedly — that it sampled the latitude bands much more effectively than did the single point model. This was a useful first step, but we felt that it was imperative to investigate Capland trajectories in a more general way. Guided by the analysis of Meert et al. (2003), we have modeled large numbers of drift paths as random walks on a sphere.

\section{Random Walk Simulations}

We construct drift histories by first placing the centre (C) of Capland at a random point on the Earth's surface. Next, we choose an azimuth at random and move $\mathrm{C}$ along this direction by an angular distance $\alpha$. The complete drift path is built up by simply repeating the process, choosing a new (random) azimuth at each successive position of $\mathrm{C}$. The time interval between successive positions of $\mathrm{C}$ can be regarded as the time between major changes in plate motions, which we take to be $100 \mathrm{Myr}$. For convenience,

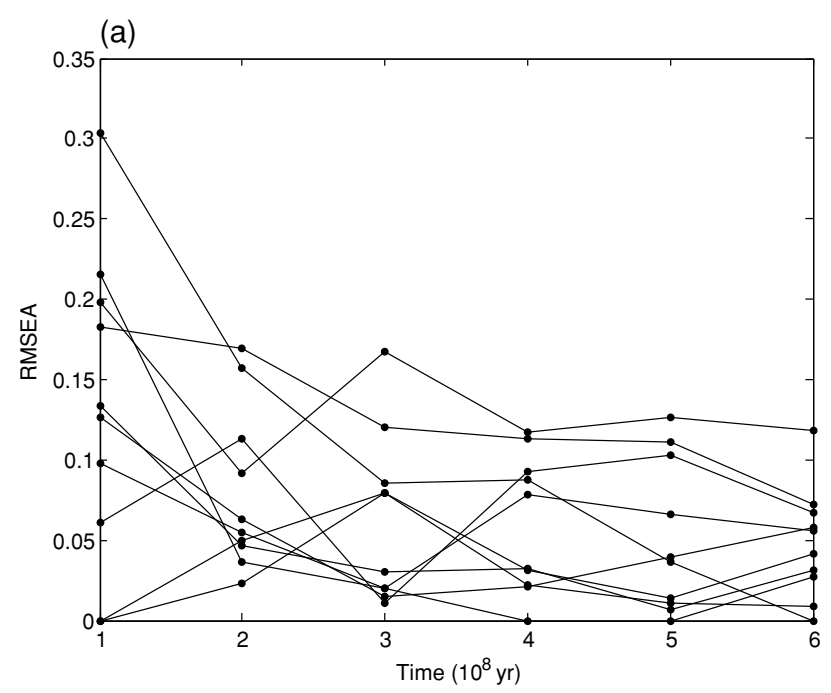

(b)

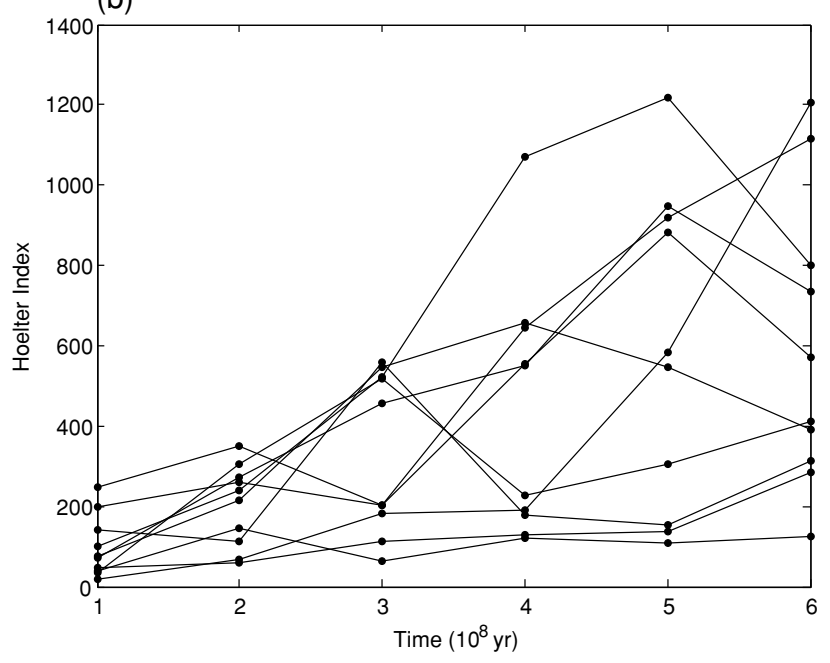

Fig. 3. (a) Root mean square error of approximation (RMSEA) as a function of time for 10 random walks as described in the text. (b) Hoelter Index $\left(N_{\text {crit }}\right)$ as a function of time for 10 random walks as described in the text.

we fix $\alpha$ at $50^{\circ}$ giving $\mathrm{C}$ a constant speed of $5.6 \mathrm{~cm} / \mathrm{yr}$. The Phanerozoic is thus modeled as a sequence of 6 positions of Capland. At each position, we mimic the combined efforts of the world's palaeomagnetists by "sampling" at N (=100) "sites" randomly distributed over the surface of Capland. The latitudes of the "sites" are binned into $10^{\circ}$ latitude intervals. Then Capland is moved to its next position and the process repeated.

Figure 2 shows an example of the latitudinal distribution of the "sampling sites" obtained from 10 separate $600 \mathrm{Myr}$ drift histories. Visually, the agreement appears satisfactory. For a more objective assessment, we calculated the two statistical parameters recommended by Meert et al. (2003): the root mean square error of approximation (RMSEA) (Nevitt and Hancock, 2000) and the Hoelter index, $N_{\text {crit }}$ (Hoelter, 1983). These parameters are related to $\chi^{2}$, but do not suffer from difficulties encountered with $\chi^{2}$ when large numbers of observations are involved. Of the ten random walks illustrated in Fig. 2, nine have RMSEA $<0.08$ and can be regarded as having adequately sampled the globe. All ten have Hoelter index values greater than 200 and can also be 


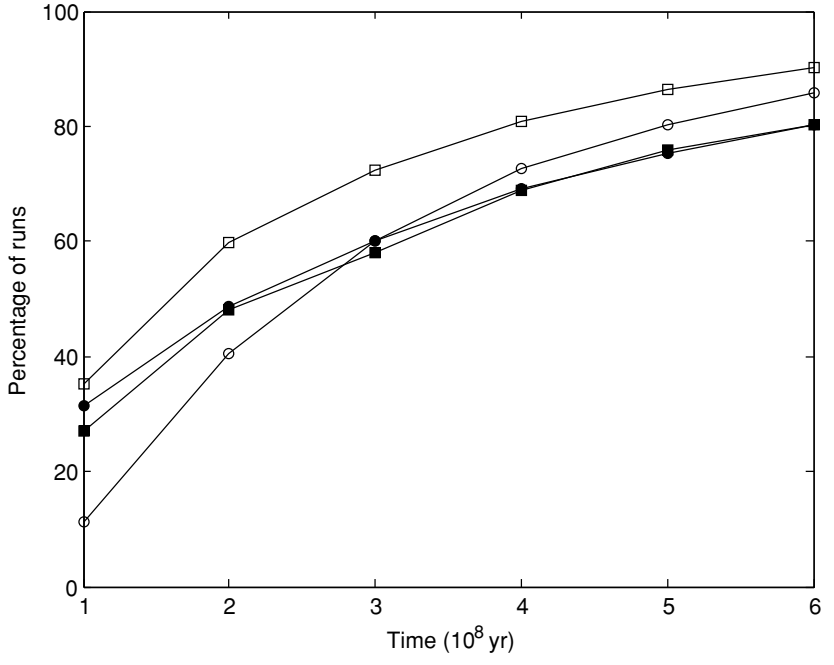

Fig. 4. Percentage of acceptable RMSEA (solid symbols) and Hoelter index (open symbols) values as a function of time. Each curve is based on 10000 separate random walks. For both statistical parameters, circles represent N=100 "sampling sites" for each position of Capland, and squares represent $\mathrm{N}=1000$.

regarded as providing adequate global coverage.

Figure 3 shows the temporal evolution of the two statistical parameters as drift takes place (using 10 new random walks). After $600 \mathrm{Myr}$, nine out of ten yield adequate fits (RMSEA $\left.<0.08, N_{\text {crit }}>200\right)$. The Hoelter index tends to continuously improve, but RMSEA has a tendency to fall rapidly and then level off, although individual drift histories show a wide variety of behaviours. Nevertheless, it is worth noting, for example, that seven out of ten cases (for both parameters) give adequate fits after only $300 \mathrm{Myr}$.

For a more thorough analysis, we ran 10000 independent drift histories, each with a different starting point produced by a random-number generator. The results are summarized in Fig. 4.

After $600 \mathrm{Myr}, 80 \%$ of the RMSEA values and $86 \%$ of the $N_{\text {crit }}$ values indicated that adequate sampling of the Earth's surface had taken place. In fact, $60 \%$ (for both parameters) are acceptable after only $300 \mathrm{Myr}$. To check the robustness of this result, we ran a second set of 10000 independent drift histories and obtained the same results, to 3 significant figures. We also investigated the sensitivity of the results to different values of $\mathrm{N}$. In order to be conservative, we have so far maintained a fixed value of $\mathrm{N}=100$, giving a total of 600 "sampling sites" for each complete drift history. This is a rather small number compared to modern global databases. We therefore repeated the entire process-using 10000 new runs each time-with $\mathrm{N}=200$, 400 and 1000. In Fig. 4, the $\mathrm{N}=1000$ results are compared to those for $\mathrm{N}=100$ (the $\mathrm{N}=200$ and 400 curves fall in between and are omitted for the sake of clarity). Evidently, RMSEA is very insensitive to sampling density, but the number of runs yielding acceptable Hoelter index values improves from $86 \%$ to $90 \%$.

\section{Discussion and Conclusions}

The fact that a large drifting continent more effectively samples the surface than does a single moving point is per- haps not surprising. But the discrepancies between our results and those of Meert et al. (2003) are more problematic. Their model leads to a success rate of only $30 \%$ as opposed to the $80 \%$ or more that we obtain, even though our random walk procedure is very similar to theirs. The reasons for this are by no means clear. Perhaps it is due to the initial biasrecognized by Meert et al. themselves-inherited from the fixed starting configurations they use (i.e. the spin axis for their "Cap World", the equator for "Equator World", and the present distribution of continents for "Today World"). As they put it, "the endpoint distribution at $600 \mathrm{Myr}$ most often reflects the initial bias". Our procedure avoids this bias by using a random number generator to establish the starting point of each run.

On the basis of large numbers of independent runs of our simple spherical cap random walk model, we conclude that 600 Myr will generally be sufficient to provide an adequate sampling of the Earth's surface. Indeed, shorter intervals may often suffice.

In closing, we stress that no claim is made that the (unique) plate tectonic history of the Earth actually did provide an adequate sampling of the globe. For example, if continents have any tendency to reside in particular latitude bands, then palaeoinclination distributions will have a corresponding bias and a resulting ambiguity (Kent and Smethurst, 1998). Our sole purpose here has been to show that, given typical plate speeds, the palaeoinclination test works in principle. If it did not-and bearing in mind the shortcomings of the other two tests (palaeoclimate, palaeointensity) discussed above - there would appear to be no means at all of testing the long-term morphology of the geomagnetic field, regardless of the specific model chosen: pure dipole, pure multipole, or, indeed, any combination of zonal harmonics.

Acknowledgments. Financial support from the Natural Sciences and Engineering Research Council of Canada is gratefully acknowledged. We thank G. J. Meert and an anonymous reviewer for their constructive comments and many useful suggestions.

\section{References}

Blackett, P. M. S., Comparisons of ancient climate with the ancient latitude deduced from rock magnetic measurements, Proc. Roy. Soc. London A, 263, 1-30, 1961.

Blackett, P. M. S., Paleomagnetism and paleoclimatology, J. Geomag. Geoelec., 13, 127-132, 1962.

Bloxham, J., Sensitivity of the geomagnetic axial dipole to thermal coremantle interactions, Nature, 405, 63-65, 2000.

Briden, J. C. and E. Irving, Paleoclimatic spectra of sedimentary paleoclimatic indicators, in Problems in Paleoclimatology, Edited by A. E. M. Nairn, pp. 199-250, Wiley Interscience, New York, 1964.

Briden, J. C., A. G. Smith, and J. T. Sallomy, The geomagnetic field in Permo-Triassic time, Geophys. J. Roy. Astron. Soc., 23, 101-117, 1970.

Evans, M. E., Test of the dipolar nature of the geomagnetic field throughout Phanerozoic time, Nature, 262, 676-677, 1976.

Evans, M. E., Testing the geomagnetic dipole hypothesis: palaeolatitudes sampled by large continents, Geophys. J. Int., 161, 266-267, 2005.

Hoelter, J. W., The analysis of covariance structures, Soc. Methods Res., 11, 325-344, 1983.

Hoffman, P. F. and D. P. Schrag, The snowball Earth hypothesis: testing the limits of global change, Terra Nova, 14, 129-155, 2002.

Irving, E., Palaeomagnetic and palaeoclimatological aspects of polar wandering, Geofis. Pura Appl., 33, 23-41, 1956.

Kent, D. V. and M. A. Smethurst, Shallow bias of paleomagnetic inclinations in the Paleozoic and Precambrian, Earth Planet. Sci. Lett., 160, 391-402, 1998. 
Korte, M. and C. G. Constable, Continuous geomagnetic field models for the past 7 millennia: 2. CALS7K, Geochemistry, Geophysics, Geosystems, 6(2), doi:10.1029/2004GC000801, 2005.

McElhinny, M. W. and W. E. Senanayake, Variations of the geomagnetic dipole I: The past 50,000 years, J. Geomag. Geoelec., 34, 39-51, 1982.

McElhinny, M. W., P. L. McFadden, and R. T. Merrill, The time-averaged paleomagnetic field 0-5 Ma, J. Geophys. Res., 101(B11), 25007-25028, 10.1029/96JB01911, 1996.

McFadden, P. L., Is 600 Myr long enough for the random palaeogeographic test of the geomagnetic axial dipole assumption?, Geophys. J. Int., 158, 443-445, 2004.

Meert, G. J., E. Tamrat, and J. Spearman, Non-dipole fields and inclination bias: Insights from a random walk analysis, Earth Planet. Sci. Lett., 214, 395-408, 2003.

Nevitt, J. and G. R. Hancock, Improving the root mean square error of approximation for nonnormal conditions in structural equation modeling, J. Exp. Educ., 68, 251-268, 2000.

Perrin, M. and V. Shcherbakov, Paleointensity of the Earth's magnetic field for the past $400 \mathrm{Ma}$ : Evidence for a dipole structure during the Mesozoic low, J. Geomag. Geoelect., 49, 601-614, 1997.
Piper, J. D. A. and S. Grant, A palaeomagnetic test of the axial dipole assumption and implications for continental distribution throughout geological time, Phys. Earth Planet. Inter., 55, 37-53, 1989.

Runcorn, S. K., The Earth's Core, Trans. Amer. Geophys. Union, 53, 4963, 1954.

Runcorn, S. K., On the hypothesis that the mean geomagnetic field for parts of geological time has been that of a geocentric axial multipole, $J$. Atmos. Terr. Phys., 14, 167-174, 1959.

Tanaka, H., M. Kono, and H. Uchimura, Some global features of palaeointensity in geological time, Geophys. J. Int., 120, 97-102, 1995.

Torsvik, T. H. and R. van der Voo, Refining Gondwana and Pangea palaeogeography; estimates of Phanerozoic non-dipole (octupole) fields, Geophys. J. Int., 151, 771-794, 2002.

Westphal, M., Did a large departure from the geocentric axial dipole field occur during the Eocene? Evidence from the magnetic polar wander path of Eurasia, Earth Planet. Sci. Lett., 117, 15-28, 1993.

Wilson, R. L., Palaeomagnetism in Northern Ireland, Geophys. J. Roy. Astron. Soc., 5, 45-58, 1961.

M. E. Evans (e-mail: evans@phys.ualberta.ca) and G. S. Hoye 